\title{
Unified Method for the Prediction of Rain Attenuation in Satellite and Terrestrial Links
}

\author{
L. da Silva Mello, Marlene S. Pontes \\ Pontifical Catholic University of Rio de Janeiro Rio de Janeiro, Brazil \\ R. Mqs. S. Vicente, 225 - Rio de Janeiro -22453-100 - RJ, Brazil - smello@cetuc.puc-rio.br
}

\begin{abstract}
In this paper, a semi-empirical method for the prediction of rain attenuation in slant paths and terrestrial links is proposed. The method uses the same simplified model of equivalent rain cell that is the basis for the ITU-R rain attenuation prediction methods but, additionally, the concept of an effective rain rate is introduced. This allows the use of the full rainfall rate distribution for the prediction of the rain attenuation distribution and the unification of the slant path and terrestrial links prediction algorithms. The numerical coefficients in the method's expressions were derived by multiple non-linear regressions using the experimental data currently available in the ITU-R data banks. Test results indicate that the proposed method provides significant improvement over the current ITU-R methods.
\end{abstract}

Index Terms-rain attenuation, propagation modeling, terrestrial links, satellite links.

\section{INTRODUCTION}

The methods for the prediction of rain attenuation in slant path links and terrestrial links currently recommended by the ITU-R are based on simplified models for the rain field affecting the propagation path. The basic assumption in these methods is that an equivalent cell of uniform rainfall rate can model the non-uniform rainfall along the propagation path.

The terrestrial prediction method, given in Rec. ITU-R P.530-13 [1], assumes that an equivalent cylindrical cell of uniform rain can intercept the link at any position with equal probability. An effective path length is calculated as the average length of the intersection between the cell and the propagation path. As a result of these assumptions the effective path length is always smaller than the actual path length, leading to the definition of a path reduction factor. In the slant path prediction method, given in Rec. ITU-R P.618-12 [2], both a horizontal and a vertical reduction factors are employed to take into account the spatial and temporal variability of the rain field.

One shortcoming of the current ITU-R methods is that the attenuation is predicted using only the measured rainfall rate exceed at $0.01 \%$ of the time. For other percentages of time, the attenuation is obtained using an extrapolation function. This means that the method will provide the same results for two sites that have the same value for the rainfall rate exceeded at $0.01 \%$ of the time, no matter if their rainfall rate distributions only coincide at this point and not for other time percentages. Another important limitation is that, when tested against the experimental data now available, the terrestrial 
prediction method shows significant underestimation of the measured attenuations. Also, the slant path and terrestrial prediction methods are not consistent, as different expressions for the horizontal path reduction factor are used in each case.

An alternative, semi-empirical method for the prediction of rain attenuation in terrestrial links was recently proposed [3], which addresses the problems observed in the current ITU-R method. The concept of an effective rainfall rate was introduced, in order to avoid path correction factors larger than the unity. The numerical coefficients in the method were derived by multiple non-linear regressions using the experimental data of rain attenuation in terrestrial links currently available in the ITU-R data banks.

In the present paper, the method proposed for terrestrial links is extended for slant path links, by the introduction of a vertical path reduction factor. The expression for this factor is derived using the experimental data on rain attenuation in slant path links currently available in the ITU-R data bank. The horizontal path reduction factor previously obtained from terrestrial measurements is kept, what ensures consistence between the slant path and terrestrial links cases.

\section{PREDICTION OF RAIN ATTENUATION IN TERRESTRIAL LINKS}

\section{A. Attenuation Due to Precipitation}

Rain attenuation is the major propagation impairment for systems operating at frequencies above 10 GHz. The presence of hydrometeors, particularly rain, in the propagation path causes scattering and absorption of the propagating wave. The raindrops behave as dissipative dielectric media to the incident wave. The scattering is associated with modifications of wave propagation directions to satisfy boundary conditions at the raindrops surfaces. The combination of these two effects causes attenuation, which depends the drops conductivity and shape.

The specific attenuation for uniform rain $\gamma_{R}(\mathrm{~dB} / \mathrm{km})$ at a given frequency may be obtained from the knowledge of the complex index of refraction of water at the temperature of the raindrops, the terminal velocity and the size distribution of the raindrops [3], [4], [5]. Due to the non-spherical shape of the falling raindrops, horizontally polarized waves suffer greater attenuation than vertically polarized waves [6], [7]. For practical applications, the relationship between specific attenuation $\gamma_{R}$ $(\mathrm{dB} / \mathrm{km})$ and rain rate $\mathrm{R}(\mathrm{mm} / \mathrm{h})$ can be approximated by a power-law $\gamma_{\mathrm{R}}=\mathrm{k} \mathrm{R}^{\alpha}$ [8]. The recent work carried out by Gibbins and Walden [9] is the basis for Recommendation ITU-R P.838-3 [10] that provides values for the coefficients $\mathrm{k}$ and $\alpha$ as functions of frequency, $\mathrm{f}(\mathrm{GHz})$, in the range from 1 to $1000 \mathrm{GHz}$. These functions have been developed as curve-fittings to power-law coefficients derived from scattering calculations.

If the rainfall rate variation along a given path is known, the attenuation due to rainfall along the path may be calculated by integrating the specific attenuation over the path length. The field of 
rainfall rate is inhomogeneous in space and time [11]. Rain gauge records show short intervals of higher rain rate imbedded in longer periods of lighter rain. Weather radar observations show small areas of higher rain rate imbedded in larger regions of lighter rain [12], [13]. Such observations are typical of all occurrences of rain in all climate regions. Rainfall is often described as widespread or stratiform and as convective, but the differences between these types usually lie in the maximum rain rate to be associated with the rain process and not in differences in spatial variability.

The main difference in the various methods developed for predicting rain attenuation statistics from rainfall rate measurements is in the models used to describe the time-space structure of rainfall rate. The synthetic storm method generates attenuation statistics by converting rain rate/time profiles recorded at a point to rain rate/distance profiles, using the translation velocity of the rain pattern, that is estimated as the wind speed [14], [15], [16]. Recently, there has been extensive work being carried out in time series synthesizers to provide synthesized rain attenuation time series [18].

All other methods make use of cumulative distributions of rainfall rate measured at a point as input to predict the attenuation. Some methods derive the statistical profile of rain along the path assuming a single cell of suitable shape [19], or a statistical distribution of sizes for cells of a particular shape [20], [21], [22]. Other methods characterize the statistical rain profile simply by a reduction coefficient, which may be derived from the spatial correlation function of rainfall, from measurements using rapid response rain gauges spaced along a line [23] or from a semi-empirical law.

An alternative procedure is to apply the reduction coefficient to the actual path length, which yields an effective path length over which the rain intensity may be assumed to be constant [24], [25], [26], [27]. This concept of an effective path length, to take into account the non-uniform profile of rain intensity along a given path in the prediction of the rain attenuation cumulative distribution on radio links, is presently used in attenuation prediction methods such as that in Recommendation ITU-R P.530-13 [1].

\section{B. ITU-R prediction method}

The method for the prediction of rain attenuation in terrestrial links, given in Recommendation ITU-R P.530-13 [1], was originally developed based on a simplified model for the temporal and spatial random variations of rain field causes the attenuation. The basic assumption in the method is that an equivalent cell of uniform rainfall rate and length $\mathrm{d}_{0}$, randomly positioned in the great circle plane, can represent the effect of the non-uniform rainfall along the propagation path.

Assuming that this equivalent rain cell may intercept the link at any position with equal probability, the expression for an effective path length is calculated. The effective path length is the average length of the intersection between the cell and path, given by:

$$
\mathrm{d}_{\text {eff }}=\langle\mathrm{L}\rangle=\frac{1}{\mathrm{~d}_{0}+\mathrm{d}} \int_{-\mathrm{d}_{0}}^{\mathrm{d}} \mathrm{L}(\mathrm{x}) \mathrm{dx}=\mathrm{r} \cdot \mathrm{d}=\frac{1}{1+\mathrm{d} / \mathrm{d}_{0}} \cdot \mathrm{d}
$$


The variables involved in the calculation are indicated in Fig. 1.

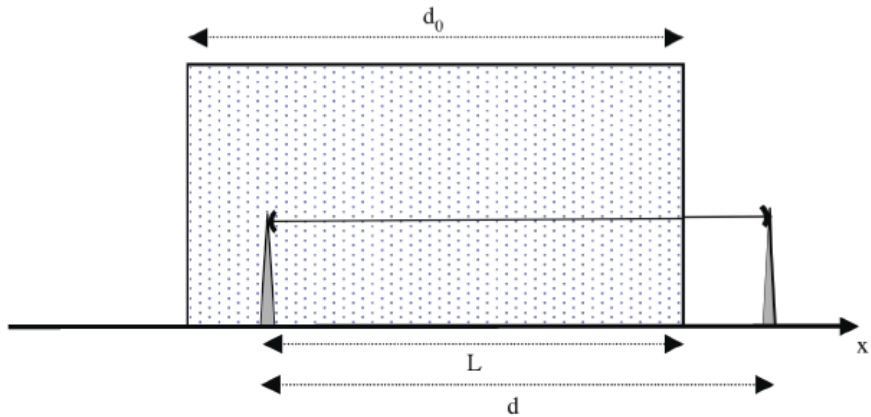

Fig. 1: Equivalent rain cell

The diameter $\mathrm{d}_{0}$ of the equivalent cell is empirically derived from experimental data, depending on the long-term point rainfall rate measured in the region. In the current recommended model, $\mathrm{d}_{0}$ is obtained from the long-term complementary cumulative probability distribution of the point rainfall rate $R(\mathrm{~mm} / \mathrm{h})$ measured in the link region. The rainfall rate exceeded at $0.01 \%$ of time $\left(\mathrm{R}_{0.01}\right)$ is used to predict the corresponding value of rain attenuation $\left(\mathrm{A}_{0.01}\right)$

$$
\mathrm{A}_{0.01}=\gamma_{0.01} \cdot \mathrm{d}_{\mathrm{eff}}=\mathrm{k}\left(\mathrm{R}_{0.01}\right)^{\alpha} \cdot \frac{\mathrm{d}}{1+\mathrm{d} / \mathrm{d}_{0}\left(\mathrm{R}_{0.01}\right)}
$$

where $\gamma(\mathrm{dB} / \mathrm{km})$ is the specific attenuation, calculated using the frequency and polarization dependent parameters k and $\alpha$, given in Recommendation ITU-R P. 838-3 [10], and $\mathrm{d}$ is the actual path length.

To calculate the attenuation exceeded at other percentages of time between $1 \%$ and $0.001 \%$ an extrapolation formula is used [1]. This represents a shortcoming of the method, as in two regions with different distributions of point rainfall rate but similar values of $\mathrm{R}_{0.01}$ the same behavior for the attenuation will be predicted. Also, empirical evidence [28] based on measured data now available indicates that the current model parameters, adjusted with the little data existing several years ago, may lead to significant underestimation of rain attenuation, particularly for tropical regions with severe rain regimes.

Some attempts to modify the ITU-R method and improve its accuracy have recently been made [29], [30], [31], [32]. It is usually found that, to correct the underestimation simply by refitting the method against the larger database of experimental data now available, it would be necessary to allow for effective path lengths longer than the actual path length. However, as it can be seen from (1), the effective path length $d_{\text {eff }}$ is always smaller than the actual path length $d$, leading to the definition of a path reduction factor $r=d_{\text {eff }} / d_{0}$.

\section{Modified prediction method for terrestrial links}

A modified method has been proposed [33] that addresses some of the problems found in the current ITU-R method but retains the general expression for $\mathrm{d}_{\mathrm{eff}}$, which is the basis of the model, and 
uses the full rainfall rate distribution at the links region as input for the prediction of the cumulative distribution of rain attenuation.

As a starting point, the dependence of the reduction factor on link parameters was investigated, using experimental data from concurrent long-term measurements of point rainfall rate and rain attenuation in terrestrial links available in the ITU-R databanks [34]. A correction factor $r_{p}$ was calculated not only for $0.01 \%$ of time, but for all percentages of time for which data is available, using

$$
r_{p}=\frac{A_{p}}{k R_{p}^{\alpha} \cdot d}
$$

where $A_{p}$ and $R_{p}$ are the rain attenuation and the point rainfall rate exceeded at $p \%$ of the time, respectively. It was found that $r_{p}$ decreases with the path length and the point rainfall rate, as depicted in Figs. 2 and 3.

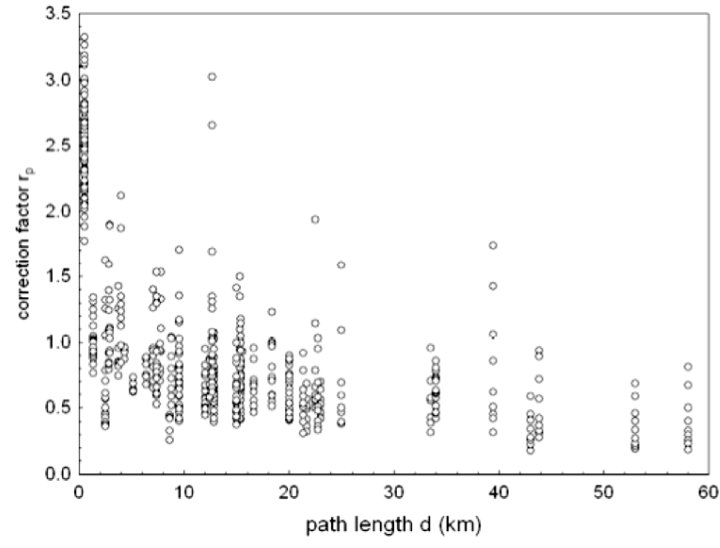

Fig. 2. Correction factor vs. path length

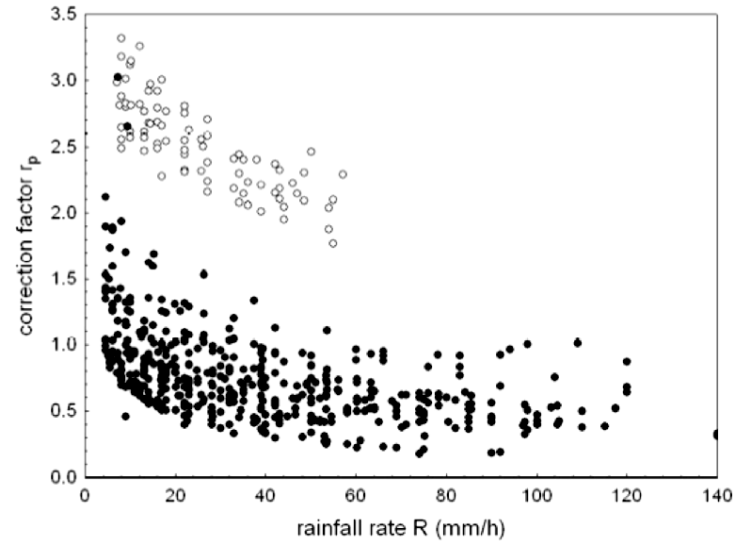

Fig. 3. Correction factor vs. rainfall rate $\mathrm{d}>1 \mathrm{~km}$ (solid) and $\mathrm{d}<1 \mathrm{~km}$ (hollow)

In Fig. 3, a very distinct behavior is observed for links shorter than $1 \mathrm{~km}$, that explains why it is necessary to allow for correction factors larger than 1 to improve the methods accuracy by refitting the current ITU-R method.

To avoid inconsistencies and retain the general expression for $d_{\text {eff }}$ given by (1), the concept of an effective rainfall rate was introduced. The cumulative distribution of rain attenuation is obtained from the distribution of rainfall rate in the links region by

$$
A_{p}=\gamma_{p} \cdot d_{\text {eff }}=k\left[\left(R_{e f f T}\left(R_{p}, d\right)\right]^{\alpha} \cdot \frac{d}{1+d / d_{0}\left(R_{p}\right)}\right.
$$

where $R_{\text {effT }}$ is the effective rain rate for terrestrial links. The empirical expression obtained for this effective rainfall rate in given by (5). The behavior of $R_{\text {effT }}$ with $R$ for different values of $d$ is shown in Fig. 4. 


$$
\mathrm{R}_{\text {effT }}=1.763 \mathrm{R}^{0.753+0.197 / \mathrm{d}}
$$

For the equivalent cell diameter $\mathrm{d}_{0}$, it was found that a power-law could provide better results than the exponential law used in the current ITU-R method. The expression obtained is given in (6). The behavior of $d_{\text {eff }}$ with $R$, for different values of $d$, is shown in Fig. 5 .

$$
\mathrm{d}_{0}=119 \cdot \mathrm{R}^{-0.244}
$$

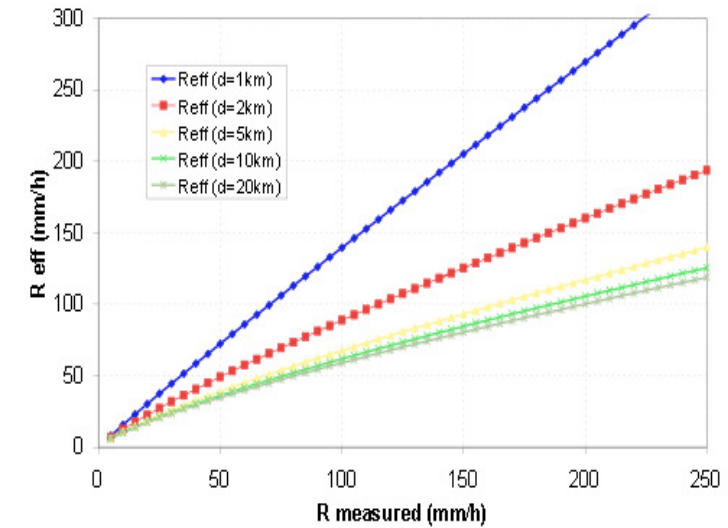

Fig. 4. Effective rainfall rate (terrestrial links)

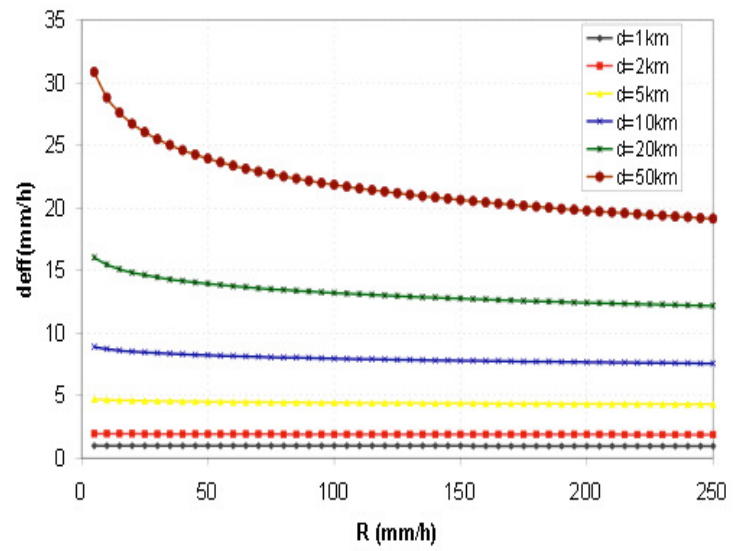

Fig. 5. Effective path length (terrestrial links)

\section{UNIFIED METHOD FOR SLANT PATHS AND TERRESTRIAL LINKS}

\section{A. Effective path length for slant paths}

The model for the effective path length can be extended for the slant path case by considering the rain height. The rain height is defined as a function of the zero degree isotherm height, which is mapped all over the world and given in Rec. ITU-R P.839-3 [35]. For a slant path with an elevation angle $\theta$, the effective path length will be given by

$$
\mathrm{L}_{\text {eff }}=\langle\mathrm{L}\rangle=\frac{1}{\mathrm{~L}_{0}+\mathrm{L}_{\mathrm{S}} \cos \theta} \int_{-\mathrm{L}_{0}}^{\mathrm{L}_{\mathrm{S}} \cos \theta} \mathrm{L}_{\mathrm{S}}(\mathrm{x}) \mathrm{dx}=\frac{1}{1+\frac{\mathrm{L}_{\mathrm{s}} \cos \theta}{\mathrm{L}_{0}}} \cdot \mathrm{L}_{\mathrm{S}}
$$

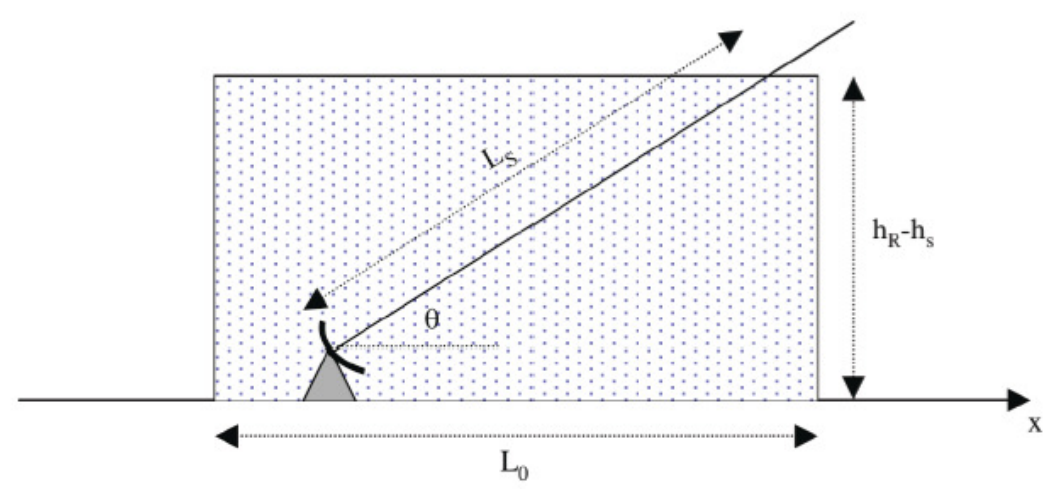

Fig. 6. Equivalent rain cell for slant paths 


\section{B. General method for rain attenuation prediction}

To obtain a more general prediction method that includes the slant path case but is still consistent with the terrestrial case, the rain attenuation cumulative probability distribution can be calculated by

$$
A_{p}=\gamma_{p} \cdot L_{e f f}=k\left[\left(R_{e f f}\left(R_{p}, L_{S}, \theta\right)\right]^{\alpha} \cdot \frac{L_{S}}{1+L_{S} \cos \theta / L_{0}}\right.
$$

For the slant path case $L_{S}=\left(h_{R}-h_{S}\right) / \sin \theta$, where $h_{R}$ is the rain height, $h_{S}$ is the antenna height above mean sea level and $\theta$ is the elevation angle. For the terrestrial case, the elevation equals zero, $\mathrm{L}_{0}$ $=\mathrm{d}_{0}$ and $\mathrm{L}_{\mathrm{S}}$ becomes the terrestrial path length $\mathrm{d}$.

The dependence of the effective rain rate on link parameters was investigated, using experimental data from concurrent long-term measurements of point rainfall rate and rain attenuation in slant path links available in the ITU-R databanks [10]. Only data from beacon measurements (not data from radiometer measurements) with concurrent measurements of rainfall rate were considered. The values of $\mathrm{R}_{\text {eff }}$ were obtained from the measured distribution of attenuation and rainfall rate by

$$
\mathrm{R}_{\mathrm{eff}}=\left(\frac{\mathrm{A}_{\mathrm{p}}}{\mathrm{k} \cdot \frac{\mathrm{L}_{\mathrm{S}}}{1+\mathrm{L}_{\mathrm{s}} \cos \theta / \mathrm{L}_{0}}}\right)^{1 / \alpha}
$$

The dependence of the effective rainfall rate with the point rainfall rate, the slant path length the elevation angle and the rain height found in the experimental data is shown in Figs. 7 to 10.

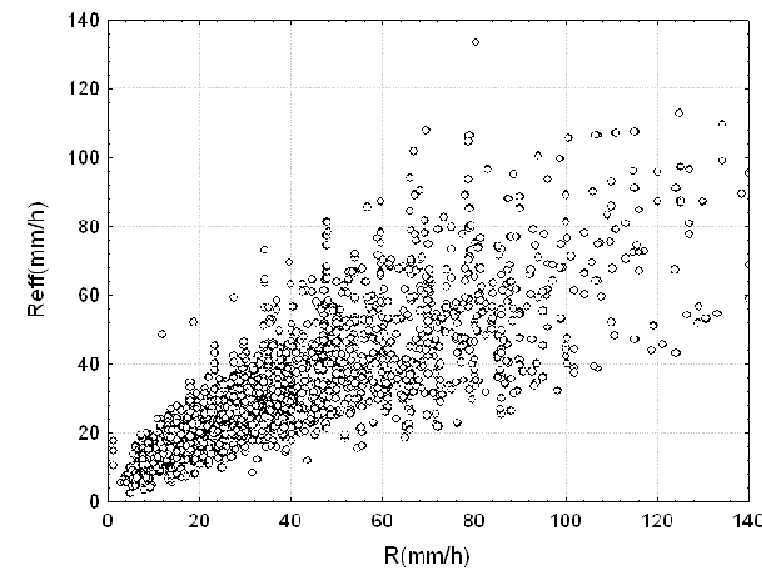

Fig. 7. Effective rain rate vs. point rainfall rate

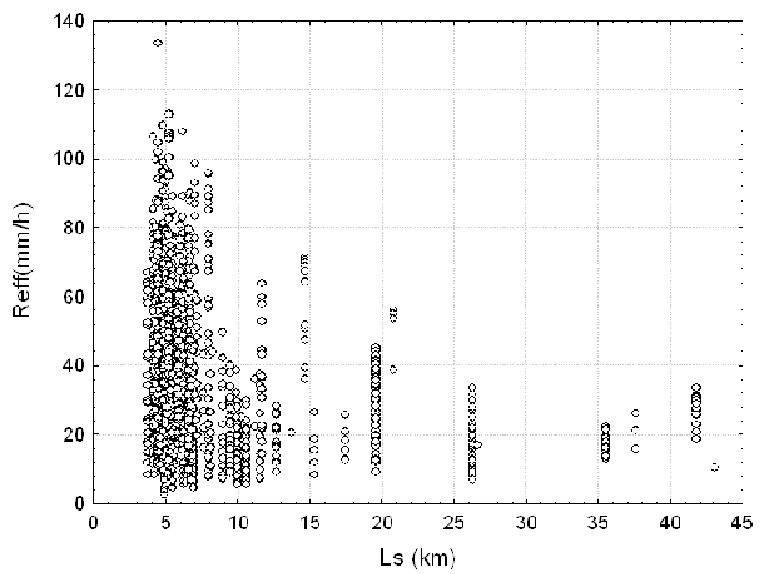

Fig. 8. Effective rain rate vs. slant path length 


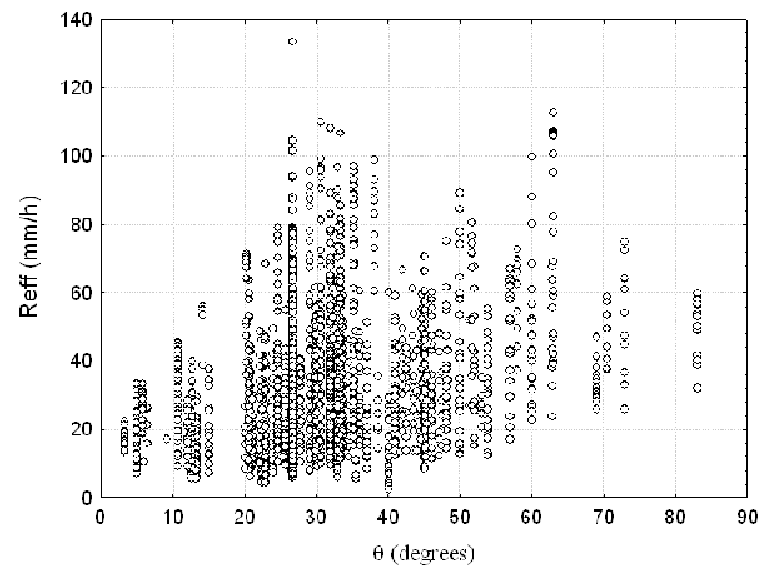

Fig. 9. Effective rain rate vs. elevation angle

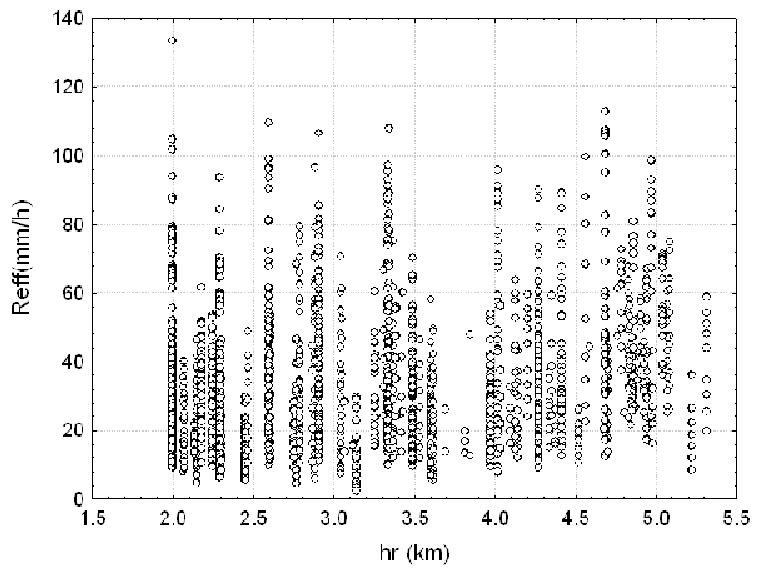

Fig. 10. Effective rain rate vs. rain height

It can be observed in Fig. 7 that the effective rain rate is strongly dependent of the point rainfall rate as should be expected. Although a larger scatter is observed in Figs. 8 and 9, it also decreases with the slant path length and shows a moderate increase with the elevation angle. Even considering that the attenuation dependence with these two variables is weaker, it is expected from a physical point of view and they were included in the method. On the other hand, the dependence on the rain height was found to be too weak for this variable to be considering in modeling.

Based on these observations, and after a series of trials with different functions, the following expression was chosen to fit $\mathrm{R}_{\mathrm{eff}}$.

$$
\mathrm{R}_{\text {eff }}\left(\mathrm{R}_{\mathrm{p}}, \mathrm{L}_{\mathrm{S}}, \theta\right)=\mathrm{R}_{\text {effT }}\left(\cos \theta+\mathrm{a}_{1} \cdot \mathrm{R}^{\mathrm{a}_{2}+\mathrm{a}_{3} / \mathrm{L}_{\mathrm{S}} \cos \theta} \cdot \mathrm{L}_{\mathrm{S}}^{\mathrm{a}_{4}} \cdot \sin \theta\right)
$$

The combination of two terms depending on $\cos \theta$ and $\sin \theta$ was used to ensure the consistence with the terrestrial case. Fitting this expression to the values obtained from the experimental data provided the values for $\mathrm{a}_{1}$ to $\mathrm{a}_{4}$. From (8) and (10), with $\mathrm{R}_{\text {effT }}$ given by (5) and $\mathrm{L}_{0}=\mathrm{d}_{0}$ given by (6), the general expression for rain attenuation prediction is

$$
\mathrm{A}_{\mathrm{p}}=\mathrm{k}\left[1.763 \mathrm{R}^{0.753+0.197 / \mathrm{L}_{\mathrm{S}} \cos \theta} \cos \theta+\frac{203.6}{\mathrm{~L}_{\mathrm{S}}^{2.455}} \mathrm{R}^{0.354+0.088 / \mathrm{L}_{\mathrm{S}} \cos \theta} \sin \theta\right]^{\alpha} \frac{\mathrm{L}_{\mathrm{S}}}{1+\frac{\mathrm{L}_{\mathrm{s}} \cos \theta}{119 \mathrm{R}^{-0.244}}}
$$

For the terrestrial case $\mathrm{L}_{S}=\mathrm{d}$, the second term in brackets vanishes $(\theta=0)$ and the expression reduces to

$$
\mathrm{A}_{\mathrm{p}}=\mathrm{k}\left[1.763 \mathrm{R}^{0.753+0.197 / \mathrm{L}_{\mathrm{S}} \cos \theta}\right]^{\alpha} \frac{\mathrm{d}}{1+\frac{\mathrm{d}}{119 \mathrm{R}^{-0.244}}}
$$

\section{COMPARATIVE TESTS OF PREDICTION METHODS}

The proposed method was tested against the ITU-R methods and other methods proposed in the technical literature, using the test variable recommended by the ITU-R [36]. This test variable is the weighted natural logarithm of the ratio between the predicted and measured values of the attenuation exceeded at a given percentage of time. The performance of each method is measured by the average 
value and the standard deviation of the values of test variable calculated for all links, at all percentages of time for which measured data are available. The test procedure adopted was the one recommended by the ITU-R, which is detailed described in [36].

For the terrestrial case, the data used to test the prediction methods includes concurrent measurements of rainfall rate, analyzed with one-minute integration time, and rain attenuation available in the ITU-R databank [34], a total of 74 year-stations from 64 links in 15 countries. The tests included the proposed method, the current ITU-R method [1], the Australian method [29], the China method [31] and the UK method [32]. Table I shows the average values, standard deviations and the r.m.s. values of the test variable for each method, which are depicted in Figs. 11 to 13.

\section{A. Tests with terrestrial links}

The test results indicate that, for the terrestrial case, the proposed method provides a large improvement over the method currently recommended by the ITU-R. The only other method that provides similar results is the China's method, which uses one single point of the rainfall rate distribution to predict the attenuation distribution. The path reduction factor and the extrapolation function used in this method show frequency dependency that should be restricted to $\mathrm{k}$ and $\alpha$.

TABLE I. PREDICTION ERROR -TERRESTRIAL LINKS

\begin{tabular}{cccccccccccccccccc}
\hline & \multicolumn{4}{c}{ Average value } & \multicolumn{4}{c}{ Standard deviation } & \multicolumn{4}{c}{ r.m.s. value } \\
\hline $\mathrm{p}(\%)$ & ITU-R & UK & AUS & China & Prop. & ITU-R & UK & AUS & China & Prop. ITU-R & UK & AUS & China Prop. \\
\hline 0.001 & -0.11 & -0.11 & 0.07 & 0.04 & 0.09 & 0.37 & 0.37 & 0.44 & 0.24 & 0.23 & 0.38 & 0.38 & 0.44 & 0.24 & 0.24 \\
0.002 & -0.13 & -0.11 & 0.06 & 0.03 & 0.06 & 0.35 & 0.36 & 0.42 & 0.22 & 0.22 & 0.37 & 0.38 & 0.43 & 0.22 & 0.23 \\
0.003 & -0.16 & -0.14 & 0.05 & 0.02 & 0.03 & 0.34 & 0.36 & 0.43 & 0.21 & 0.21 & 0.38 & 0.38 & 0.43 & 0.21 & 0.21 \\
0.006 & -0.17 & -0.13 & 0.05 & 0.01 & 0.01 & 0.35 & 0.34 & 0.42 & 0.20 & 0.21 & 0.39 & 0.37 & 0.43 & 0.20 & 0.21 \\
0.01 & -0.17 & -0.12 & 0.05 & 0.00 & 0.01 & 0.36 & 0.35 & 0.44 & 0.20 & 0.22 & 0.40 & 0.37 & 0.44 & 0.20 & 0.22 \\
0.02 & -0.20 & -0.13 & 0.03 & -0.02 & -0.02 & 0.38 & 0.37 & 0.44 & 0.23 & 0.25 & 0.43 & 0.39 & 0.44 & 0.23 & 0.25 \\
0.03 & -0.22 & -0.13 & 0.02 & -0.03 & -0.04 & 0.40 & 0.37 & 0.46 & 0.25 & 0.26 & 0.46 & 0.40 & 0.46 & 0.25 & 0.26 \\
0.06 & -0.27 & -0.18 & -0.04 & -0.05 & -0.09 & 0.43 & 0.36 & 0.47 & 0.27 & 0.27 & 0.51 & 0.41 & 0.47 & 0.28 & 0.29 \\
0.1 & -0.32 & -0.24 & -0.10 & -0.15 & -0.12 & 0.45 & 0.36 & 0.49 & 0.31 & 0.30 & 0.55 & 0.43 & 0.49 & 0.34 & 0.32 \\
\hline
\end{tabular}




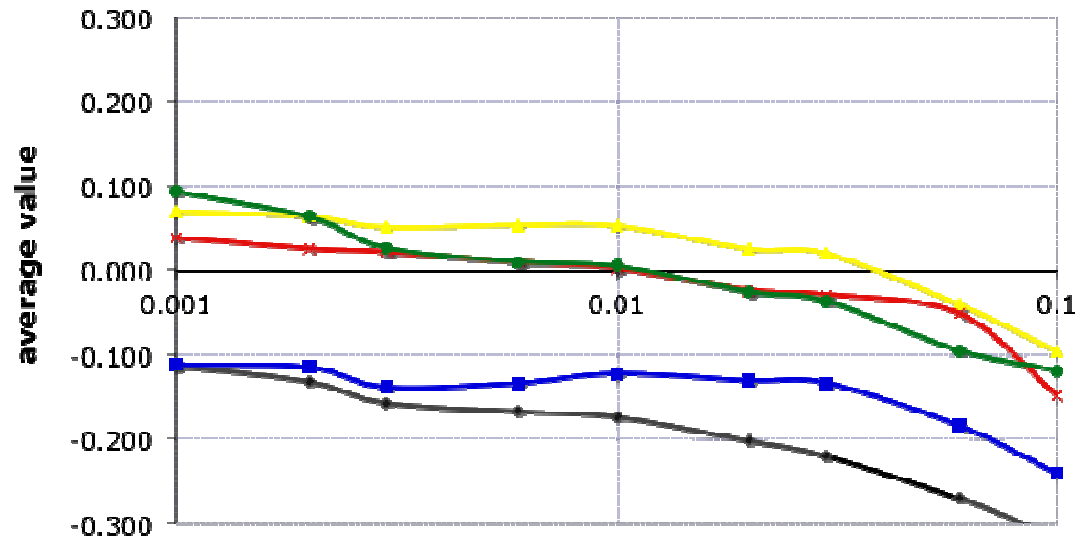

percentage of time

-Rec. ITU-R P.530-13 -UJK - Australia *China -proposed

Fig. 11. Average error - terrestrial links

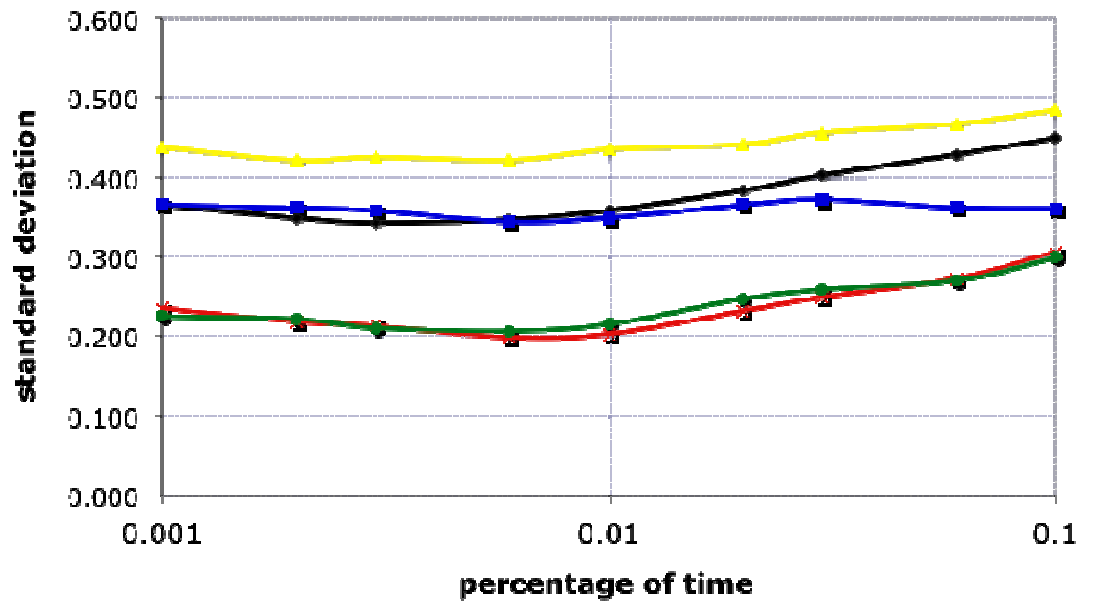

-Rec. ITU-R P.530-13 -UK Australia - China -proposed

Fig. 12. Standard deviation - terrestrial links

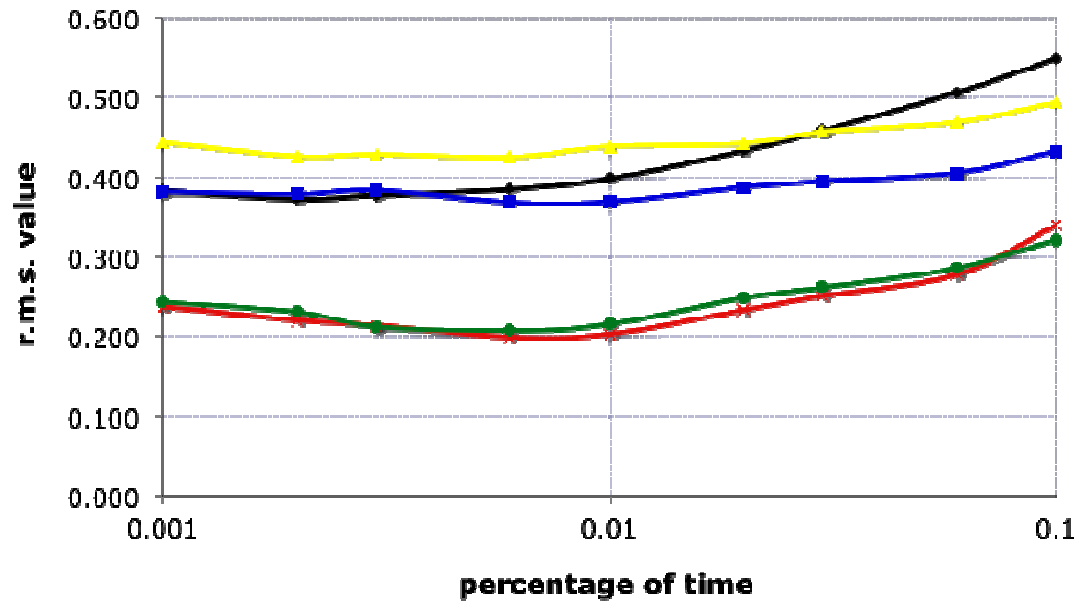

$\rightarrow$ Rec. ITU-R P.530-13 $\rightarrow$-UK $\rightarrow$ Australia $*$ China $\rightarrow$ proposed

Fig. 13. R.m.s value - terrestrial links 


\section{B. Tests with satellite links}

For the slant path case, the data used to test the prediction methods includes concurrent measurements of rainfall rate and rain attenuation in received satellite beacon signals, also available in the ITU-R databank [34], comprising a total of 280 year-stations from 68 sites in 24 countries. The tests included the proposed method, the current ITU-R method [2], the Australian method [38], the China method [39] and the UK method [40]. Table II and Figs. 14 to 16 show the average values, standard deviations and the r.m.s. values of the test variable for the slant path links.

TABLE II. PREDICTION ERROR - SLANT PATH LINKS

\begin{tabular}{cccccccccccccccccc}
\hline \multicolumn{1}{c}{ Average value } & \multicolumn{1}{c}{ Standard deviation } & \multicolumn{4}{c}{ r.m.s. value } \\
\hline $\mathrm{p}(\%)$ & ITU-R & UK & AUS & China & Prop. & ITU-R & UK & AUS & China & Prop. & ITU-R & UK & AUS & China & Prop. \\
0.001 & 0.06 & -0.05 & 0.02 & 0.08 & 0.004 & 0.32 & 0.32 & 0.31 & 0.34 & 0.31 & 0.33 & 0.32 & 0.31 & 0.35 & 0.31 \\
0.002 & 0.00 & -0.05 & -0.01 & 0.00 & -0.01 & 0.33 & 0.31 & 0.33 & 0.34 & 0.30 & 0.33 & 0.32 & 0.33 & 0.34 & 0.30 \\
0.003 & -0.03 & -0.05 & -0.04 & -0.04 & -0.03 & 0.31 & 0.30 & 0.31 & 0.32 & 0.29 & 0.32 & 0.31 & 0.32 & 0.32 & 0.29 \\
0.005 & -0.06 & -0.05 & -0.07 & -0.06 & -0.04 & 0.29 & 0.30 & 0.32 & 0.29 & 0.28 & 0.29 & 0.30 & 0.32 & 0.30 & 0.28 \\
0.010 & -0.08 & -0.03 & -0.07 & -0.07 & -0.03 & 0.29 & 0.29 & 0.30 & 0.29 & 0.28 & 0.30 & 0.29 & 0.31 & 0.30 & 0.28 \\
0.020 & -0.08 & 0.00 & -0.11 & -0.05 & -0.02 & 0.27 & 0.27 & 0.30 & 0.27 & 0.27 & 0.28 & 0.27 & 0.32 & 0.27 & 0.27 \\
0.030 & -0.08 & 0.03 & -0.12 & -0.02 & 0.00 & 0.26 & 0.27 & 0.29 & 0.26 & 0.27 & 0.27 & 0.27 & 0.31 & 0.26 & 0.27 \\
0.050 & -0.08 & 0.04 & -0.15 & 0.01 & 0.01 & 0.25 & 0.27 & 0.31 & 0.26 & 0.27 & 0.26 & 0.27 & 0.34 & 0.26 & 0.27 \\
0.100 & -0.10 & 0.05 & -0.20 & 0.02 & 0.03 & 0.28 & 0.30 & 0.36 & 0.28 & 0.30 & 0.30 & 0.30 & 0.41 & 0.28 & 0.30 \\
\hline
\end{tabular}

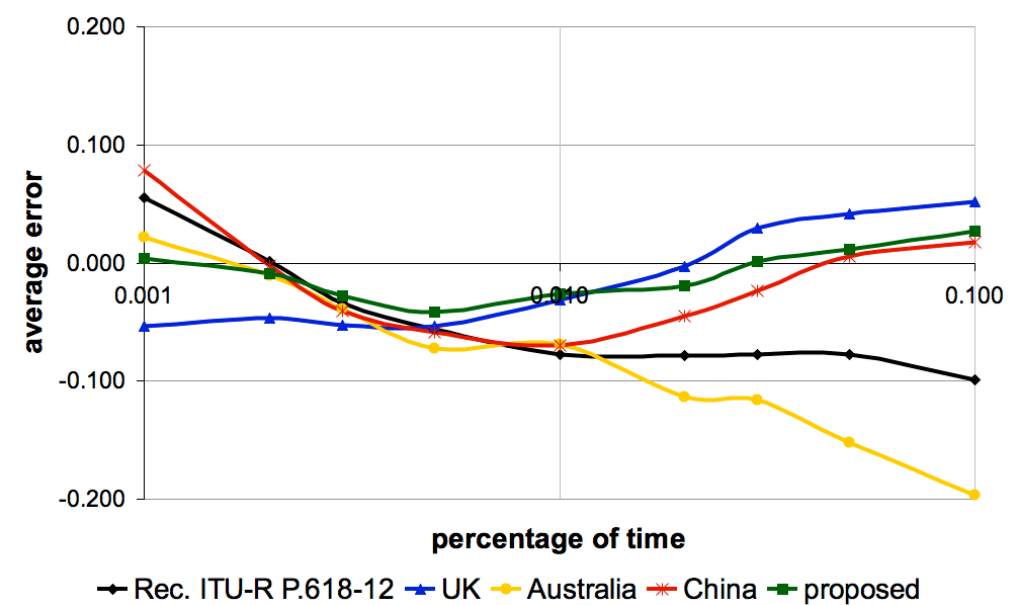

Fig. 14. Average error - slant path links 


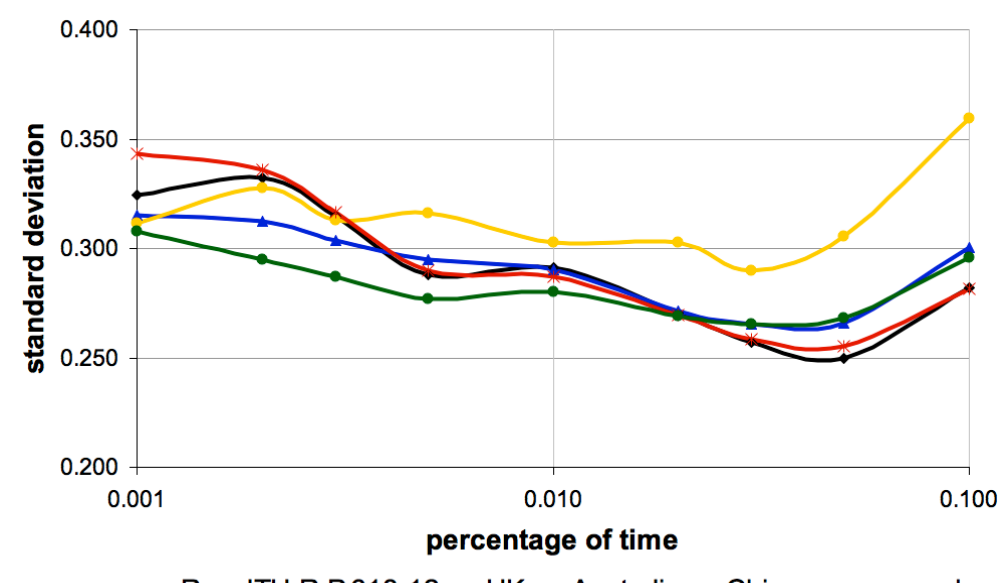

$\rightarrow$ Rec. ITU-R P.618-12 $\rightarrow$ UK $\rightarrow$ Australia * China $\rightarrow$ proposed

Fig. 15. Standard deviation - slant path links

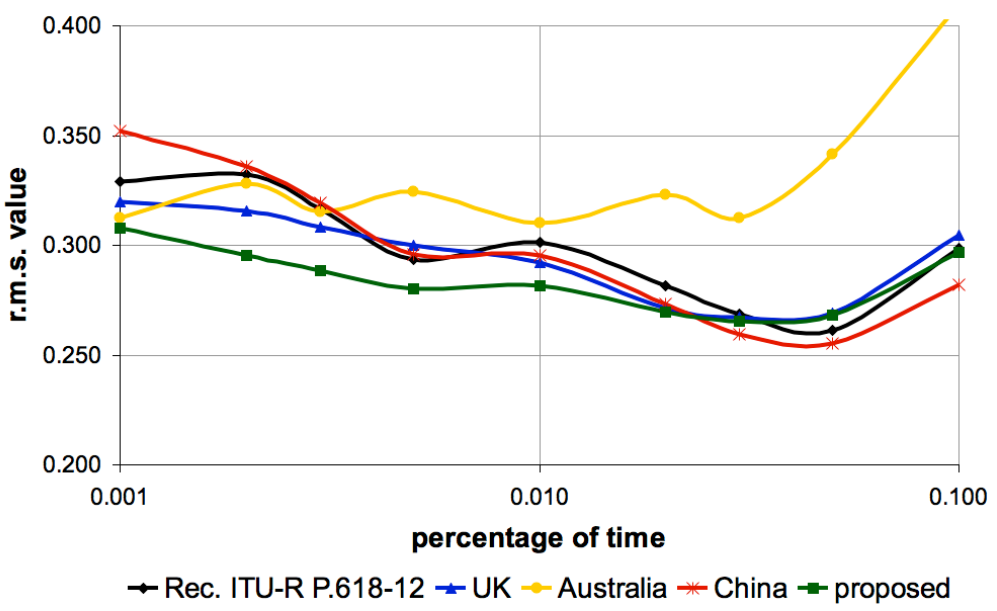

Fig. 16. R.m.s value - slant path links

The test results indicate that, for low percentages of time, the proposed method provides a significant improvement over the method currently recommended by the ITU-R. For percentages of time between 0.03 and $0.1 \%$ the two methods are approximately equivalent. In this time percentage range, the China's method provides slightly better results than the other methods.

\section{CONCLUSIONS}

The new method proposed for the prediction of rain attenuation in terrestrial and slant path is simple to apply and uses the full rainfall rate distribution to predict the attenuation distribution, avoiding the extrapolations dependent on the percentage of time.

The concept of an equivalent rain cell, which is the basis of the original ITU-R methods, is retained in the new method and the attenuation dependence on frequency is completely described by the parameters $\mathrm{k}$ and $\alpha$, as should be expected from the physical point of view. Consistency between the terrestrial and the slant path cases was also achieved, which is not present in any of the methods tested.

Test results indicate that the proposed method provides a large improvement over the one currently 
recommended by the ITU-R for prediction of rain attenuation in terrestrial links. The performance is essentially equivalent to that of the method proposed by China, which presents inconsistencies from the physical point of view.

For earth-space links, a significant improvement is also obtained for low percentages of time. In the time percentage range bellow $2 \times 10^{-2} \%$ the unified method shows better results than any of the methods considered here.

\section{REFERENCES}

[1] Recommendation ITU-R P. 530-13, "Propagation data and prediction methods required for the design of terrestrial lineof-sight systems", International Telecommunication Union, 2009.

[2] ITU-R Recommendation P.618-12: 'Propagation data and prediction methods required for the design of Earth-space systems', International Telecommunication Union, 2006.

[3] J.W. Ryde and D. Ryde, "Attenuation of Centimetre and Millimetre Waves by Rain, Hail, Fog, and Clouds", Rpt. No. 8670, Research Laboratory of the General Electric Company, Wembley, England.

[4] R. Medhurst, "Rainfall attenuation of centimeter waves: Comparison of theory and measurement", IEEE Trans. Ant. Prop., pp. 550- 564.

[5] J.E. Setzer, "Computed transmission through rain at microwave and visual frequencies", Bell System Tech. J., vol. 49, pp.1873-1892.

[6] T. Oguchi and Y. Hosoya, "Differential attenuation and differential phase shift of radio waves due to rain: Calculations of microwave and millimeter wave regions", J. Rech. Atmos., vol. 8, pp. 121-128.

[7] T. Oguchi, "Scattering properties of Pruppacher-and-Pitter form raindrops and cross-polarization due to rain: calculations at 11,13, 19-3 and 34-8 GHz", Radio Sci., 12, pp. 41-51.

[8] R. Olsen, D. Rogers, and D. Hodge, "The aRb relation in the calculation of rain attenuation", IEEE Trans. Ant. Prop., vol. 26, no. 2, pp. 318- 329.

[9] C.J. Gibbins, C.J. Walden, "A study into the derivation of improved rain attenuation regression coefficients", Radicommunications Agency Report No. AY4359, 2003, http://www.radio.gov.uk/topics/research/research-index.htm.

[10] Recommendation ITU-R P. 838-3, "Specific attenuation model for rain for use in prediction methods".

[11] S.A. Callaghan and E. Vilar, "The spatial distribution of rain and its implications for wide area communications systems", ICAP 2003, Exeter.

[12] C. Capsoni, M. D'Amico, "Morphological description of the rain structures in the Padana Valley", Proceedings of the Third European Conference on Radar Meteorology (ERAD), pp. 541-544, Sweden, September 2004.

[13] C. Capsoni, L. Luini, A. Paraboni and C. Riva, "Stratiform and convective rain discrimination deduced from local P(R)", IEEE Transactions on Antennas and Propagation, vol. 54, no. 11, pp. 3566-3569, November 2006.

[14] G. Drufuca, "Rain attenuation statistics for frequencies above $10 \mathrm{GHz}$ from rain gauge observations", Journal Recherches Atmospheriques, vol 1-2, pp. 399-411, 1974.

[15] H.N. Kheirallah, B. Segal and R.L. Olsen, "Application of Synthetic Storm Data to Evaluate Simpler Techniques for Predicting Rain Attenuation Statistics", Ann. Télécommunic, vol. 35, no. 11-12, pp. 456-462, 1980.

[16] E. Matricciani, and C. Riva, "Test of the probability formulation of the Synthetic Storm Technique against reliable measurements of rain rate and rain attenuation", Antennas and Propagation Symposium, 2008.

[17] E. Matricciani, "Global formulation of the Synthetic Storm Technique to calculate rain attenuation only from rain rate probability distributions", Antennas and Propagation Symposium, 2008.

[18] F. Lacoste, M. Bousquet, L. Castanet, F. Cornet, J. Lemorton, "Improvement of the ONERA-CNES rain attenuation time series synthesizer and validation of the dynamic characteristics of the generated fade events", Space Communication Journal, Vol. 20, $n^{\circ} 1-2,2005$

[19] P. Misme and J. Fimbel, "Theoretical and experimental determination of rain-induced attenuation on a radioelectric path", Annales des Telecommunications, vol. 30, pp. 149-158, 1975.

[20] C. Capsoni, F. Fedi, C. Magistroni, A. Paraboni and A. Pawlina, "Data and theory for a new model of the horizontal structure of rain cells for propagation applications", Radio Sci., vol. 22, no. 3, pp. 395-404, May-June 1987.

[21] C. Capsoni, F. Fedi, A. Paraboni, “A comprehensive meteorologically oriented methodology for the prediction of wave propagation parameters in telecommunication applications beyond $10 \mathrm{GHz}$ ", Radio Science, vol. 22, no. 3, pp. 387-393, May-June 1987.

[22] A. Paraboni, G. Masini, and A. Elia, "The Effect of Precipitation on Microwave LMDS Networks - Performance Analysis using a Physical Raincell Model”, IEEE J. on Selected Areas in Commun., vol. 20, no. 3, pp. 615-619, April 2002 .

[23] B.N. Harden, J. Norbury and A. White, "Use of a lognormal distribution of raindrop sizes in millimetric radio attenuation studies", IEE Conf. Publ. 169, part 2, pp. 87-91, 1978.

[24] R.K. Crane, "Prediction of attenuation by rain", IEEE Trans. Commun., vol. 28, pp. 1717-1733, 1980. 
[25] S.H. Lin, "A method for calculating rain attenuation distributions on microwave paths", Bell Syst. Tech. J., pp. 10511086,1975

[26] J.A. Garcia Lopez and J. Peiro, "Simple rain attenuation prediction technique for terrestrial radio links", Elect. Letters, vol. 19, pp. 879-881, 1983.

[27] F. Moupfouma, "Improvement of a rain attenuation prediction method for terrestrial microwave links", IEEE Transactions on Antennas and Propagation, vol. 32, pp. 1368-1372, 1984.

[28] L. A. R. Silva Mello, E. P. O. Costa, R. S. L. Souza, 'Rain attenuation measurements at 15 and $18 \mathrm{GHz}$ ', Electronics Letters, Londres, UK, v. 40, n. 11, p. 683-684, 2004.

[29] ITU-R, Study Group 3, Working Party 3M, Input Document 3M/27(Study Period 2000-2003), Australia, "Proposed amendement to Recommendation ITU-R P.530-9: Rain attenuation effective path length”, 17 May 2001.

[30] ITU-R, Study Group 3, Working Party 3M, Input Document 3M/208, Federative Republic of Brazil, "Proposed modification to Recommendation ITU-R P.530-11: Propagation data and prediction methods required for the design of terrestrial line-of-sight systems", 11 April 2007.

[31] ITU-R, Study Group 3, Working Party 3M, Input Document 3M/107, People's Republic of China, "Proposed modification to Recommendation ITU-R P.530-10: An improved prediction method of rain attenuation for terrestrial light-of-sight path”, 19 September 2005.

[32] ITU-R, Study Group 3, Working Party 3M, Input Document 3M/28, United Kingdom "Proposed modification to Recommendation ITU-R P.530-10: Propagation data and prediction methods required for the design of terrestrial lineof-sight systems", 12 November 2003.

[33] Silva Mello, L.A.R., Pontes, M.S., Souza, R. M. and Pérez-Garcia, N. A., 'Prediction of rain attenuation in terrestrial links using the full rainfall rate distribution', Electronics Letters, v. 43, p. 1442-1443, 2007.

[34] ITU-R Databank DBSG3, http://www.itu.int/pub/R-SOFT-SG3/en.

[35] ITU-R Recommendation P.839-3: 'Rain height model for prediction methods', International Telecommunication Union, 2001.

[36] ITU-R Recommendation P.311-12: 'Acquisition, presentation and analysis of data in studies of tropospheric propagation', International Telecommunication Union, 2006.

[37] ITU-R Working Party 3M (WP 3M) - Fascicles, http://www.itu.int/ITU-R/index.asp?category=studygroups\&rlink=rwp3m-fascicles\&lang=en.

[38] ITU-R, Study Group 3, Working Party 3M, Input Document 3M/134, 'Proposed modifications to Recommendation ITU-R P.618-8 - Propagation data and prediction methods required for earth-space telecommunication systems', UK.

[39] ITU-R, Study Group 3, Working Party 3M, Input Document 3M/20 (Study Period 1996-1997), 'Proposed amendment to Recommendation ITU-R PN.618 - Prediction of Rain Attenuation', Australia.

[40] ITU-R, Study Group 3, Working Party 3M, Input Document, 'Proposed modification to Recommendation ITU-R P.530-10 - The prediction method of rain attenuation along Earth-space path', China (Peoples Republic of). 